Erschienen in: Plewnia, Albrecht/Witt, Andreas (Hrsg.): Sprachverfall? Dynamik - Wandel Variation. Berlin/Boston: de Gruyter, 2014. (Jahrbuch des Instituts für Deutsche Sprache 2013), S. S. 273-298

Stefan Kleiner (Mannheim)

\title{
Die Kodifikation der deutschen Standardaussprache im Spiegel der faktischen Variabilität des Gebrauchsstandards
}

\begin{abstract}
Die Normierung der deutschen Standardaussprache geht in ihren Ursprüngen auf die 1898 durch die Siebs-Kommission beschlossenen Regelungen für die deutsche Bühnenaussprache zurück. Seit 1964 bildet der Nachrichtensprecher bei der Ausübung seines Berufs die Grundlage für die gegenwärtigen deutschen Aussprachekodizes. Diese eingeschränkte empirische Basis zusammen mit einem primär präskriptiven Anspruch der Kodifikatoren führt dazu, dass auch das aktuellste Aussprachewörterbuch des Deutschen (DAW) in vielen Fällen den tatsächlichen Standardsprachgebrauch in Deutschland nur unzureichend repräsentiert. Dies wird im vorliegenden Beitrag durch den Vergleich mit Sprachdaten aus dem Korpus „Deutsch heute“, das Lese- und Spontansprache v.a. von Oberstufenschülern am Gymnasium aus dem ganzen deutschen Sprachraum enthält, anhand von acht unterschiedlichen sprachlichen Phänomenen gezeigt. Der Beitrag schließt mit einem Plädoyer für realitätsnähere Kodifikationen, die sich am Sprachgebrauch der ,educated speaker" orientieren (wie es v.a. im englischsprachigen Raum der Fall ist), weil sie der aktuellen Sprachsituation im Deutschen weit besser Rechnung tragen als die derzeit existierenden Kodizes.
\end{abstract}

\section{Die Entstehung und Entwicklung der deutschen Aussprachenorm}

Als auslösendes Moment für die Entwicklung der deutschen Aussprachenorm wird gerne Goethe mit seinem 1803 entstandenen Zitat aus den „Regeln für Schauspieler“ als Kronzeuge angeführt, der in \$1 schreibt: „Wenn mitten in einer tragischen Rede sich ein Provinzialismus eindrängt, so wird die schönste Dichtung verunstaltet und das Gehör des Zuschauers beleidigt“" (Goethe 1982, S. 252). Es gab also „,[s]eit der Herausbildung der deutschen Nationalliteratur" (DAW, S. 9) einen wachsenden Bedarf zur Vereinheitlichung der Aussprache auf den deutschen Theaterbühnen. Allerdings sollte es dann noch bis 1898 dauern, bis mit der Tagung einer Kommission von drei Sprachwissenschaftlern und drei Theaterdirektoren (nur einer davon war aus dem Süden des deutschen Sprachraums) unter dem Vorsitz des Initiators Theodor Siebs endgültig ein verbindlicher Katalog für die Bühnenaussprache verabschiedet wurde (Siebs (Hg.) 1898).

Von Anfang an gab es aber auch Bestrebungen, diese Regelungen nicht nur für die Bühne wirksam werden zu lassen, sondern auch für das allgemeine Sprachleben, insbesondere die Schule, als verbindliche Richtschnur 
einzusetzen. Über den Allgemeinen Deutschen Sprachverein wurden zur Beurteilung dieser Erweiterung des Geltungsbereichs verschiedene Gutachten v.a. durch namhafte Sprachwissenschaftler der Zeit in Auftrag gegeben (u.a. Kluge 1899; Behaghel 1899; Paul 1899). Die Erweiterung der Bühnenaussprache auf die Schule wird darin mehrheitlich sehr kritisch beurteilt. ${ }^{1}$ Zwei Stimmen sollen hier exemplarisch zu Wort kommen. Oskar Brenner (1899, S. 179), der aus Franken stammende und damals in Würzburg lehrende Ordinarius für Deutsche Sprache, kritisiert z.B. die mangelnde Berücksichtigung süddeutscher Formen und plädiert für die Zulassung von Varianten:

So findet der Süddeutsche in den angeführten Durchschnittsformen sehr viel ihm Widerstrebendes, was sein Ohr vielleicht noch von der Bühne her erträgt, sein Mund aber sich kaum aneignen wird. [...] mein Widerspruch [würde] an manchen Stellen gegenstandslos, wenn man öfter als es geschehen, statt eines Entwederoder ein Nebeneinander gesetzt, oder eine Zwischenstufe eingeführt hätte.

Hermann Paul übt Fundamentalkritik am Ansinnen, die Aussprache zu vereinheitlichen, und hält nur eine empirisch fundierte, landschaftliche Eigenheiten berücksichtigende Normierung für sinnvoll (Paul 1899, S. 189 f.):

Auch im Schulunterricht und sogar beim Lesen, wo die Anforderungen am strengsten sein müssen, wird man nicht eine völlig andere Gewöhnung durchführen können. Der Lehrer muß in Bekämpfung der mundartlichen Eigenheiten der Aussprache ein bestimmtes Maß halten. Sonst unternimmt er etwas äußerst Schwieriges, ja fast Unmögliches und, wie ich glaube, etwas Unnützes, ja nicht einmal Wünschenswertes. [...] Es hat auch gar keinen Zweck, daß wir Deutschen alle völlig einerlei reden. Es genügt, wenn die Besonderheiten auf ein solches Maß eingeschränkt sind, daß das gegenseitige Verständnis nicht mehr behindert ist. [...] Will man für die Schulsprache bestimmte Regeln aufstellen, deren Durchführung wirklich möglich und wünschenswert ist, so muß man diesen Regeln für jede Landschaft eine besondere Fassung geben.

Trotz der Kritik wurden die Regelungen von der Versammlung deutscher Schulmänner und Philologen auch für die Schule empfohlen, was allerdings erst 1922 mit der Erweiterung des Titels der Siebs-Kodifikation um „Hochsprache“ explizit umgesetzt wurde (vgl. Siebs 1931b, S. 10).

1931 wurde der Geltungsbereich der Siebs-Norm dann ein weiteres Mal mit einer - inhaltlich nicht signifikant modifizierten - Version auf die „Rundfunkaussprache“ (Siebs 1931a) erweitert (vgl. DAW, S. 11).

Der Siebs hat schließlich in seiner 1969 erschienenen letzten Auflage eine wesentliche Überarbeitung auch dadurch erfahren, dass mit der gemäBigten Hochlautung in begrenztem Umfang auch allophonische Varianten eingeführt ${ }^{2}$ und erstmals nationale Varianten auch im Wörterverzeichnis gebucht wurden.

1 Von den insgesamt sieben Stellungnahmen fallen fünf überwiegend bis ausgesprochen negativ aus, nur zwei sind positiv.

2 Z.B. [ge]- neben [gə]- oder uvulares [R] neben alveolarem $[\mathrm{r}]$. 
Dass schon vor Siebs Wilhelm Viëtor (1885) der eigentliche Begründer der deutschen Aussprachekodizes war, ist heute kaum mehr bekannt. Teilweise sind die in seinem Aussprachewörterbuch (z.B. Viëtor 1931, Erstauflage 1908) gebuchten Formen auch als realitätsnäher einzuschätzen als die im Siebs kodifizierten.

Ab 1962 tritt auch der Duden-Verlag auf den Plan und gibt das von Max Mangold erarbeitete „Aussprachewörterbuch“ heraus. Dessen Alleinstellungsmerkmal ist ein u.a. durch die massive Erweiterung um deutsche und fremdsprachige Toponyme auf 110.000 Stichwörter angewachsenes Wörterverzeichnis. Mit der 2. Auflage (Duden 1974) übernimmt der Ausspracheduden dann wesentliche Ergebnisse aus dem 1964 erstmals (unter der Federführung von Hans Krech) in Leipzig herausgegebenen „Wörterbuch der deutschen Aussprache" (WDA), das mit dem 1982 erschienenen „Großen Wörterbuch der deutschen Aussprache“ (GWDA) vier Auflagen erlebt. Dessen große Leistung war es, erstmals mit der Beschreibung des Sprachgebrauchs von Nachrichtensprechern bei der Ausübung ihres Berufs eine konsequente empirische Basis zur Grundlage für einen Aussprachekodex zu machen. Dies führte im Vergleich zu den bisherigen Kodifikationen zu einem deutlichen Realitätsgewinn im Sinne der Annäherung der kodifizierten Formen an einen tatsächlich existierenden Sprachgebrauch.

Etwas ausführlicher soll der aktuellste Kodex besprochen werden, das „Deutsche Aussprachewörterbuch“ (DAW), das 2009 als Nachfolger des GWDA und indirekt auch des Siebs erschienen ist. ${ }^{4}$ Laut Klappentext ,gibt [es] den Standard so wieder, wie er heute von den meisten Deutschsprachigen als hochsprachliche Norm empfunden und verwendet wird“. Die Annahme, dass auch dementsprechende Untersuchungen angestellt wurden, wird allerdings enttäuscht, denn es enthält als Neuerung lediglich soziophonetische Untersuchungen zu den „Erwartungen der Hörenden an den Sprechgebrauch“ (DAW, S. 15), bleibt beim empirischen Fundament allerdings im Wesentlichen bei Nachrichtensendungen „im konventionellen Stil“ (ebd., S. 16), d.h. dem Typ Tagesschau.

Bei den gebuchten Lemmata schlägt das DAW mit 150.000 Einträgen sogar noch Duden 6, was vor allem daran liegt, dass es praktisch dessen komplette Lemmaliste übernimmt, diese aber noch um eine große Zahl an Komposita und Ableitungen erweitert.

3 Im DAW findet sich einleitend (S. 8-17) ein umfangreicher Abriss der Geschichte der deutschen Aussprachekodifikation, in der auch Viëtors Anteil gewürdigt wird. Eine Lektüre dieser sachkundigen Überblicksdarstellung ist empfehlenswert, wenn auch die oben zitierten negativen Gutachten mit keiner Silbe erwähnt werden.

4 Für eine eingehendere Diskussion als sie hier möglich ist, verweise ich auf entsprechende Rezensionen des DAW (z.B. Trouvain 2011; Kleiner 2011). 
Regionale Variation wird hingegen im DAW vollständig ausgeblendet. ${ }^{5}$ Das führt dann dazu, dass eine Aussage wie „Die Lautverbindung $[\mathrm{p}]+[\mathrm{f}]$ wird im absoluten Anlaut meist zu [f] reduziert" (DAW, S. 108) schlicht unter der Rubrik „Standardaussprache mit verringerter Artikulationspräzision" verbucht wird, statt sie als typisch mittel- und norddeutsche Variante des Gebrauchsstandards aufzufassen.

Eine sehr zu begrüßende Neuerung im DAW stellen die eigenständigen, deskriptiv orientierten und damit notwendigerweise variantenreichen Kapitel von Peter Wiesinger (2009) sowie von Walter Haas und Ingrid Hove (2009) zur Standardaussprache in Österreich und der Schweiz dar. Mit diesen „soll den Benutzern eine Norm zur Verfügung gestellt werden, welche der Sprachsituation und den Bedürfnissen der Sprechenden Rechnung trägt, indem sie sich auf den realen Sprachgebrauch stützt" (Haas/Hove 2009, S. 259). Analoges gilt auch für den Beitrag zu Österreich, in dem das Modell einer gemäßigten Plurizentrizität, d.h. auch subnationaler sprachlicher Variation, als Merkmal der Standardsprache vertreten wird (Wiesinger 2009, S. 230 f.).

Aufgrund dieser beiden Beiträge sehen sich die Herausgeber des DAW veranlasst, ein „teilweise spezielle[s] Verständnis von Standardaussprache, wie es sich für Österreich und die Schweiz herausgebildet hat" (DAW, S. 7) zu konstatieren. Dabei geht es hier sicher weniger um nationale Unterschiede als um unterschiedliche Ideologien, die im selben Buch aufeinandertreffen: eine sprechwissenschaftlich-präskriptive und eine sprachwissenschaftlichdeskriptive.

Zusammenfassend lässt sich feststellen, dass - abgesehen von den Fortschritten, die sich v.a. in der Berücksichtigung der beiden südlichen nationalen Varietäten des Deutschen im DAW manifestieren - der aktuellste deutsche Aussprachekodex für die in Deutschland gesprochene Standardsprache weiterhin einen hochgradig präskriptiven Ansatz vertritt, der den Nachrichtensprecher bei der Ausübung seines Berufs immer noch zum Maß aller Dinge erhebt ${ }^{6}$ und so viele Varianten, die von großen Teilen der Bevölkerung natürlicherweise verwendet werden, in der Regel entweder ignoriert oder auf eine Notiz im Einleitungsteil beschränkt (statt sie an den entsprechenden Stellen im Wörterverzeichnis unterzubringen).

\footnotetext{
Dies steht im Gegensatz zu allen Vorläuferwerken, die immer auch in begrenztem Umfang Aussagen zu regional üblichen Varianten gemacht haben, wenn auch manchmal nur deshalb, um diese als nicht normkonform zu brandmarken.

$6 \quad$ Damit gilt weiterhin, was Kohler $(1995$, S. 38) bereits an der Konzeption des WDA kritisiert hat: „[D]ie Verabsolutierung der über die Nachrichtensprecher gewonnenen Norm als allgemein gültige Standardaussprache des Deutschen [bedeutet] einen Rückfall in die jüngere Siebstradition und schafft trotz der empirischen Basis wieder eine Idealnorm für das Sprechen außerhalb von Nachrichtensendungen".
} 


\section{Zum Konzept „Gebrauchsstandard“}

Vor der Besprechung der Auswertungsergebnisse muss erst noch ein terminologischer und konzeptioneller Grundpfeiler unseres Projekts ${ }^{7}$ erläutert werden: Mit dem Terminus „Gebrauchsstandard“ bezeichnen wir gemäß unserem korpusbasierten Ansatz die empirisch feststellbare Sprachform, die uns in formellen Situationen (Vorlesesprache, soziobiographisches Interview) unserer Spracherhebung mit Sprecherinnen und Sprechern mit höherer Schulbildung entgegentritt. ${ }^{8}$ Unser Konzept des Gebrauchsstandards steht damit dem „standard language“-Konzept der Anglistik nahe, das sich auf die „usage of educated speakers" beruft und sich nach Barbour/Stevenson (1998) auch auf die deutsche Sprache anwenden lässt, wenn man konstatiert, dass im Deutschen Regionalität eine erheblich größere Rolle spielt als im Englischen. ${ }^{9}$ Die potenziell komplexere Varietätenkonstellation im Deutschen macht es allerdings nötig, dieses Konzept in einer sprachgebietsweiten Erhebung wie der unseren aus Vergleichbarkeitsgründen auf formelle Situationen einzuschränken.

Nicht zuletzt hat bei unserer Adaptation von „Gebrauchsstandard“ auch eine Rolle gespielt, dass es sich um einen inhaltlich neutralen Terminus handelt. Die gerade in der Germanistik ziemlich häufig zu findenden Termini „intendierter Standard“ und „Substandard“ halten wir aufgrund ihrer negativen Konnotationen für nicht geeignet: Beim ,intendieren“ schwingt ganz deutlich ein „sich bemühen, aber nicht erreichen“ mit. Und die Präfigierung mit „sub“ hat mit der weltweiten Finanzkrise durch „sub-prime“ einen weiteren Grund bekommen, für die nicht pejorativ gemeinte Beschreibung einer Sprachform besser nicht verwendet zu werden.

Aus den gemachten Ausführungen wird auch klar, dass wir dem Vorschlag von Schmidt/Herrgen (2011, S. 62), „die Standardvarietät linguistisch so abzugrenzen, wie es dem Varietätenbewusstsein naiver Sprecher/ Hörer entspricht", nicht folgen können. Einerseits bleiben bei dieser Konzeptionierung soziosituative Gebrauchskontexte und das von Ammon (1995) herausgearbeitete „soziale Kräftefeld“ ohne jegliche Berücksichtigung, ande-

IDS-Projekt „Variation des gesprochenen Deutsch“, Projektleitung: Arnulf Deppermann, Mitarbeiter: Mechthild Elstermann, Stefan Kleiner, Ralf Knöbl, Rudolf Schmidt.

8 Der Terminus stammt ursprünglich von Ulrich Ammon aus seinem Buch zu den nationalen Varietäten des Deutschen (Ammon 1995), er bezeichnet damit allerdings ausschließlich nicht kodifizierte Formen, die sich ,,aufgrund ihres Vorkommens in Modelltexten als standardsprachlich bestimmen“ lassen (Ammon 1995, S. 94).

9 „Im Vergleich zu ihrem englischen Äquivalent weist sie [die deutsche Standardsprache; Anm. S.K.] ein geringeres $\mathrm{Maß}$ an rein sozial markierter phonetischer Variation auf, zeichnet sich dafür aber auf allen Ebenen (Aussprache, Grammatik, Wortschatz) durch stärkere regionale Differenziertheit aus.“ (Barbour/Stevenson 1998, S. 147). 
rerseits macht man, wenn man das genannte Varietätenbewusstsein naiver Sprecher/Hörer z.B. wie in Kehrein (2009) über deren Bewertung regional gefärbter Sprachproben ermittelt hat, deren unreflektiertes laienlinguistisches Konzept „Hochdeutsch“ zur alleinigen Richtschnur für das eigene wissenschaftliche Konzept von „Standardsprache“.

\section{Das Korpus „Deutsch heute“}

Es folgt ein kurzer Überblick über das Sprachkorpus „Deutsch heute“, das unseren Auswertungen zugrundeliegt.

Von 2006 bis 2009 wurden im ganzen deutschen Sprachraum 670 Aufnahmen mit Oberstufenschülern an Gymnasien gemacht, je vier pro Ort. An annähernd jedem zweiten Ort wurden auch zwei Personen im Alter zwischen 50 und 60 Jahren aufgenommen, als Vergleichsgruppe v.a. zur Feststellung von potenziellem Sprachgebrauchswandel. Die wesentlichen Auswahlkriterien für beide Gruppen waren höhere Schulbildung und Ortsansässigkeit seit der Geburt, außerdem sollte mindestens ein Elternteil aus der engeren Region des Erhebungsorts stammen.

Die aufgenommenen Sprachdaten bestehen zur Hälfte aus Lesesprache (Texten, einer großen Wortliste, Minimalpaaren) und zur Hälfte aus Spontansprache, die in einem soziobiographischen Interview und - nur bei den Schülern - auch über eine Wegbeschreibungsaufgabe zwischen zwei Schülern erhoben wurde.

Die im Folgenden gezeigten Ergebnisse stammen überwiegend aus Auswertungen der vorgelesenen Wortliste. Diese und in der Endausbaustufe bis 2018 ca. 300-400 weitere Karten werden auf einer Wiki-Plattform im Internet publiziert, dem „Atlas zur Aussprache des deutschen Gebrauchsstandards" (AADG).$^{10}$

\section{Vergleich der Daten}

Im folgenden Abschnitt wird an insgesamt acht Fallbeispielen konkret gezeigt, wie sich der empirisch feststellbare Gebrauchsstandard im Korpus „Deutsch heute“ zu den Vorgaben der deutschen Aussprachekodizes, besonders des DAW, verhält.

10 Die URL ist http://prowiki.ids-mannheim.de/bin/view/AADG. Dort findet sich auch eine detailliertere Beschreibung des Korpusdesigns. 


\subsection{Aussprache von $<_{\mathrm{v}}>$ in evangelisch}

Ein klassischer, systeminhärenter Variationsfall ist die Aussprache des Buchstabens $<_{\mathrm{v}}>$. Dem Graphem entsprechen im Deutschen bekanntlich mit /f/ und /v/ zwei artikulatorisch und akustisch zwar nahe verwandte, aber phonematisch unterschiedliche Realisierungen. $\langle\mathrm{v}\rangle$ tritt häufig auch in Fremdwörtern auf, in welchen zwar eine gewisse Präferenz für die [v]-Aussprache herrscht, die aber in zahlreichen Fällen klar lexemspezifisch gesteuert ist. Das führt u.a. zu nationalen Varianten, die auch in den Kodizes beschrieben werden, wobei in Österreich im Vergleich zu Deutschland eher die stimmhafte [v]-Aussprache gebräuchlich ist (wie in Verse und Vizepräsident, vgl. Wiesinger 2009, S. 241), wohingegen in der Schweiz die stimmlose [f]-Aussprache häufiger vorkommt (wie in November und Ventil, vgl. Haas/Hove 2009, S. 275 f.). Auch für Deutschland ist in diesem Bereich im DAW in einzelnen Fällen Variation kodifiziert, z.B. finden sich bei Pulver sowohl [f] als auch [v]. (bei Kurve ist allerdings nur [v] zugelassen - Duden 6 ist da toleranter und erlaubt auch hier beide Varianten).

Exemplarisch sollen die Ergebnisse $\mathrm{zu}<_{\mathrm{v}}>$ in evangelisch präsentiert werden. Die Kodizes schreiben in diesem Wort einhellig [v] vor.

Der Korpusbefund auf Karte $1^{11}$ zeigt für Deutschland eine insgesamt klare Präferenz für die [f]-Aussprache (71\%), nur ganz im Nord(osten) und im östlichen Mitteldeutschen überwiegt der [v]-Typ. ${ }^{12}$ Außerhalb Deutschlands dominiert [v] in Luxemburg, Ostbelgien, Südtirol und Österreich (wobei in letzterem ein klarer Ost-West-Gegensatz zu erkennen ist). In der Schweiz kommen beide Varianten in einem relativ ausgewogenen Verhältnis vor.

Die kodifizierte Variante wird also in Deutschland in unserem Korpus nur von einer deutlichen Minderheit verwendet, die zwar diskontinuierliche, aber doch klar erkennbare regionale Konzentrationen bildet. Dabei ist es ungewöhnlich, dass im ansonsten ziemlich geschlossenen [f]-Gebiet manchmal ganze Ortspunkte wie Kusel (KUS) oder Kleve (KLE) (fast) komplett herausfallen.

$11 \quad$ Zur Symbolisierung auf den Sprachkarten: Die Aussprachebelege der einzelnen Sprecherinnen und Sprecher werden durch kleine Quadrate symbolisiert, die in der Regel pro Ort zu einer Vierergruppe, d.h. einem großen Quadrat, zusammengefasst sind.

12 Die tatsächliche Realisierung ist im Norden Deutschlands vorwiegend frikativisch, v.a. in Österreich auch approximantisch, d.h. ohne wesentliches Reibegeräusch. 


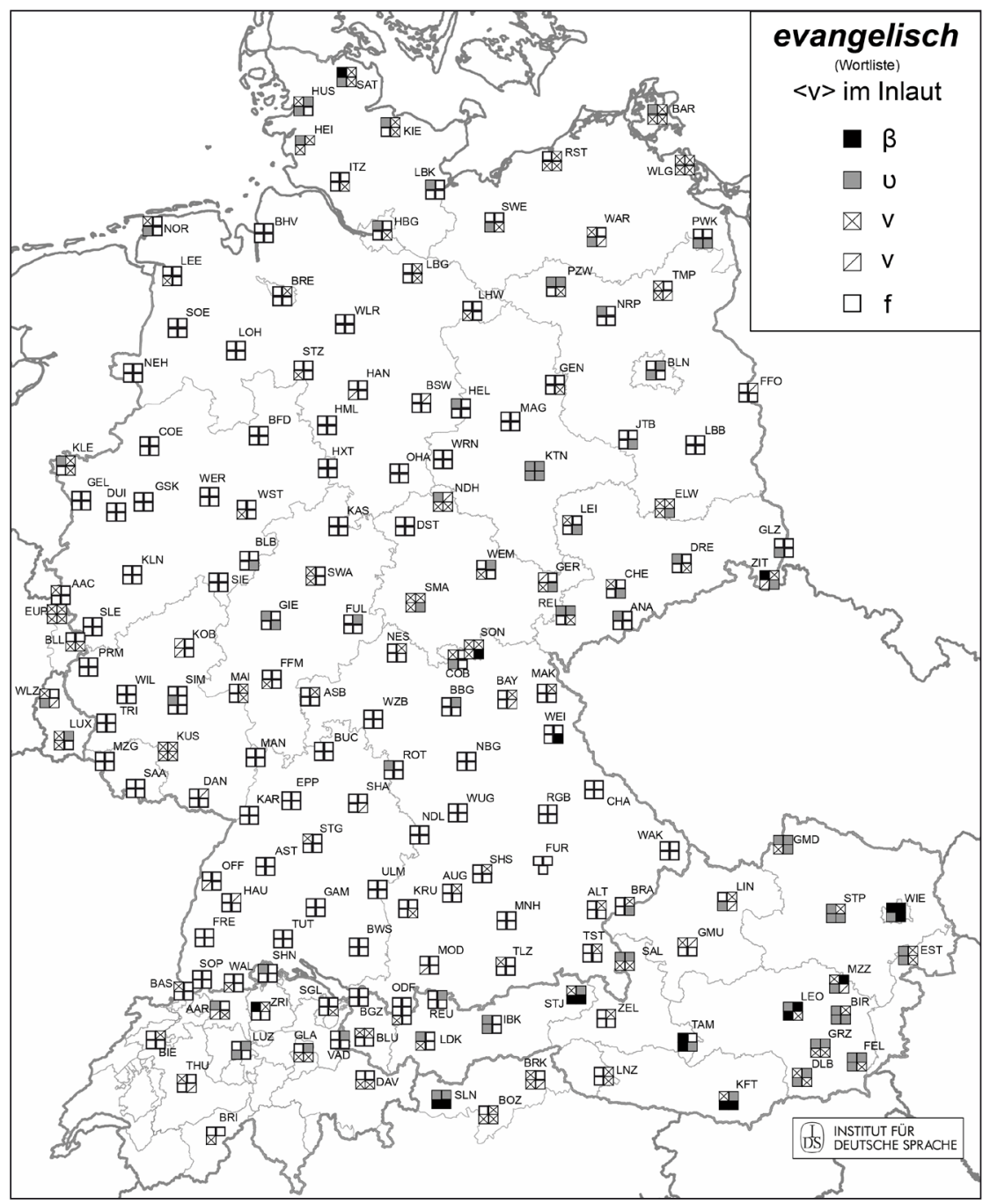

Karte 1: Aussprachevarianten für $<_{\mathrm{v}}>$ in evangelisch

\subsection{Realisierung der Morphemgrenze in Fremdwörtern}

Das zweite zu besprechende Phänomen ist komplexer, allerdings ist die dahinterstehende Problematik auch weit umfassender als die schlichte Ambiguität einer Graphem-Phonem-Korrespondenz wie bei $\left\langle_{\mathrm{v}}\right\rangle$.

Im DAW (S. 37) wird festgestellt: „Die Morphemgrenze zwischen Präfixen bzw. Halbpräfixen und dem freien Morphem ist immer auch eine Silbengrenze." Das bedeutet, morphologische Strukturen der lateinischen oder 
griechischen Wortbildung müssen auch im Deutschen beachtet werden; phonologische Prozesse, die die Morphemgrenze verundeutlichen, sind in der Regel nicht standardsprachlich und werden darum auch nicht im Wörterverzeichnis geführt. Es gibt davon nur wenige lexikalisierte Ausnahmen, zu nennen wären hier Interesse, exakt, adäquat. Sehr aufschlussreich ist aber die anschließende Feststellung:

Für viele Sprachbenutzer ist der Wortaufbau nicht immer durchsichtig. Sie setzen Sprechsilben nach deutschen Mustern an, verschieben dabei Silbengrenzen und verändern infolgedessen die Vokalqualität, z.B. wird für Deskription statt [de.skь্ııtsiino:n] dann [des.kbıiptsi'o:n] gesprochen. (DAW, S. 38)

Solche Formen sind allerdings nicht im Wörterverzeichnis des DAW gebucht, dementsprechend findet sich Mikroskop als [mikњo.sk'o:p] ganz explizit mit einem Punkt zwischen [o] und [s] transkribiert, der ausdrücklich auf die Silbengrenze zwischen den beiden Morphemen hinweist.

Mikroskop wurde auch in unserem Korpus erhoben und soll jetzt mit diesen Vorgaben verglichen werden. Der Nachweis der Silbifizierung ist allerdings nur indirekt möglich, nämlich über die Realisierung des / $\mathrm{k} /$ in [sko:p]: Wenn das / $\mathrm{k} /$ hier nicht aspiriert wird, verhält es sich, wie man es für einen deutschen Anlautcluster erwartet, in dem analog zu /sp-/, /st-/ (bzw. $/ \mathrm{Sp}-/, / \mathrm{St}-/$ ) die Aspiration fehlen sollte. Ist dem so, dann ist die Silbifizierung wie im Kodex vorgeschrieben tatsächlich auch im Gebrauchsstandard üblich. Wenn / $\mathrm{k}$ / in [sko:p] aber aspiriert wird, dann wird es realisiert wie im absoluten Anlaut, was ein deutlicher Hinweis darauf wäre, dass eine Resilbifizierung des Typs [mi.kъos.'ko:p] vorliegt.

Voraussetzung für diese Nachweismöglichkeit ist, dass / $/ \mathrm{im}$ Anlautcluster /sk-/ tatsächlich ohne Aspiration realisiert wird, was aufgrund des ziemlich peripheren Status von /sk-/ im Deutschen nicht notwendigerweise gegeben sein muss. Deswegen soll vorab erst ein unzweifelhafter Anlautcluster untersucht werden, wie er im Belegwort Skala vorliegt.

Das Ergebnis ist auf Karte 2 zu sehen: In Österreich und der Schweiz wird das / $\mathrm{k} /$ überwiegend (81\%) aspiriert/affriziert, d.h. der Nachweis auf die oben beschriebene Art und Weise ist hier nicht möglich. In Deutschland ist das / k/ allerdings zu 90\% nicht aspiriert, d.h. hier lässt sich der Nachweis führen. Nebenbei bemerkt liegt hier eine klare Tendenz zu nationalen Varianten im Deutschen vor, die bisher wohl noch nicht als solche beschrieben worden sind - was natürlich auch an der bereits angemerkten Randständigkeit des Phänomens im Deutschen liegt.

Bezüglich der Realisierung von / $\mathrm{k} /$ in Mikroskop ergibt sich für Deutschland ein zu Skala fast diametral entgegengesetztes Bild (vgl. Karte 3): 95\% der $/ \mathrm{k} /$-Belege sind aspiriert. Das bedeutet, /k/ steht in diesen Belegen im absoluten Silbenanlaut, die Resilbifizierung zu [mi.kњos.'ko:p] ist der absolute Normalfall. Zusätzlich ist auch häufig eine Vokalsenkung zu [kธ̋əs] belegt, ein weiteres Indiz für die Resilbifizierung, sie ist aber nicht obligatorisch. 


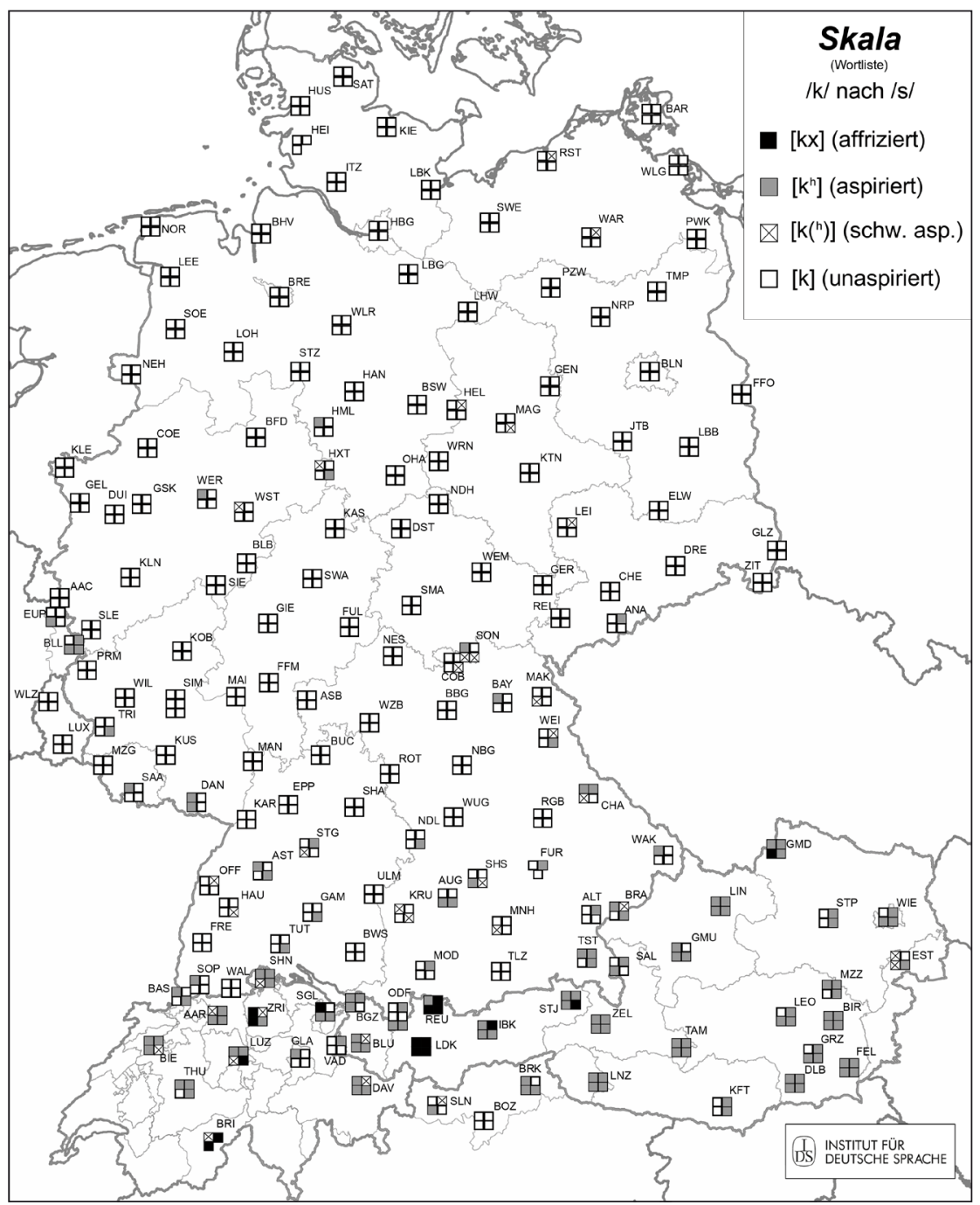

Karte 2: Aussprachevarianten für $<\mathrm{k}>$ in Skala

Das Fazit ist: Die allermeisten Belege im Korpus differieren von den Forderungen des Kodex, und man darf annehmen, dass die Gebräuchlichkeit des Lexems im Deutschen eine erhebliche Rolle dabei spielt, ob resilbifiziert wird oder nicht. 


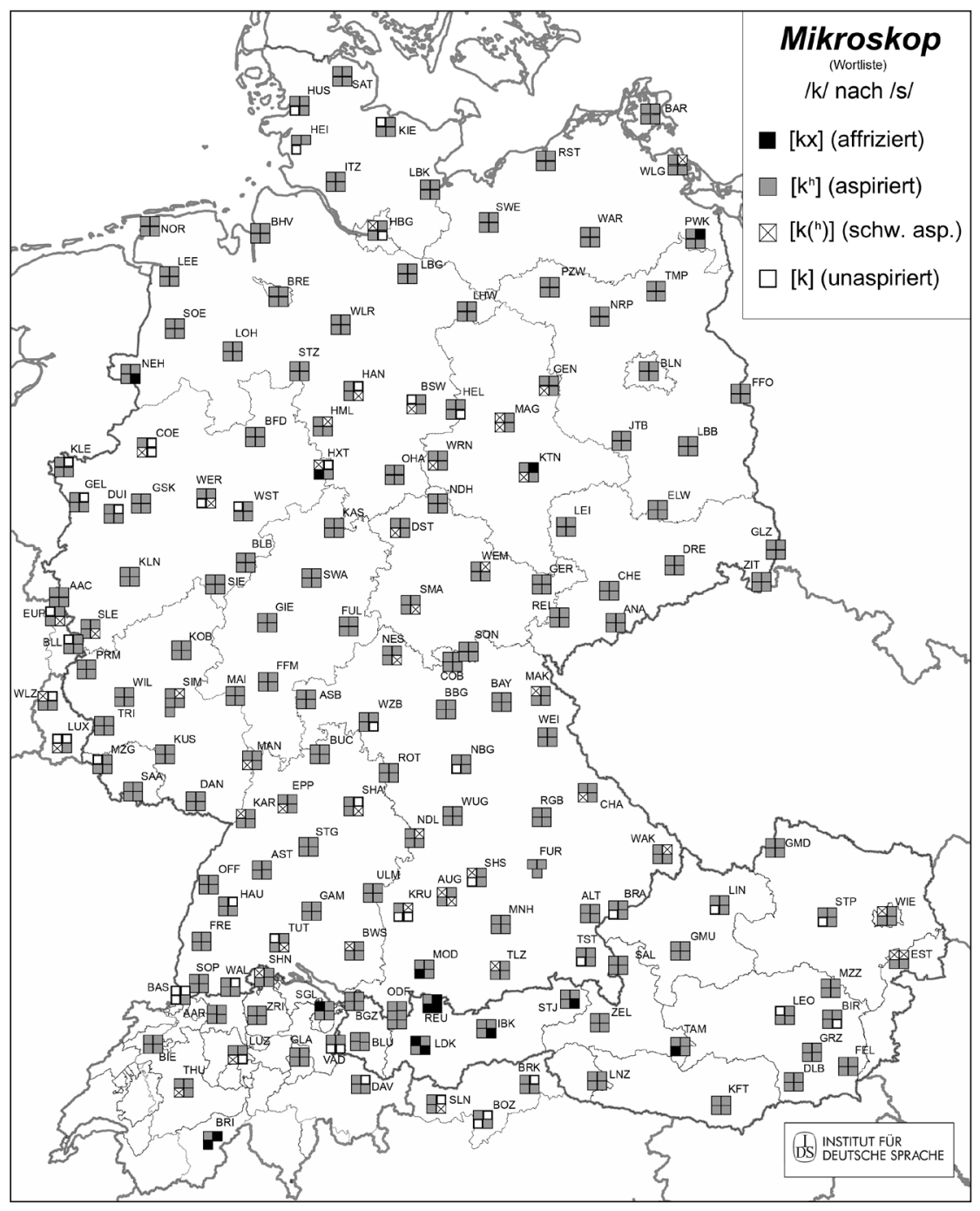

Karte 3: Aussprachevarianten für $<\mathrm{k}>$ in Mikroskop

\subsection{Glottalplosiv im Inlaut}

Auch für das jetzt zu besprechende Phänomen gibt es keine direkte graphematische Wiedergabe: Der Glottalplosiv (auch Kehlkopfknacklaut) bzw. eine diesem entsprechende Glottalisierung ist für das Deutsche im Wortanlaut vor Vokal ein sehr typisches Phänomen; hier soll jedoch die inlautende Position untersucht werden. Im DAW findet sich dazu folgendes Zitat (S. 53): 
Der Glottisschlag (Glottisplosiv) wird nicht realisiert [...] silbenanlautend in $\mathrm{Na}$ men und fremden Wörtern nach Vokal, wenn dieser nicht zu einem vokalisch anlautenden Glied in Komposita oder zu einem vokalisch auslautendem [sic!] Präfix gehört [...] aber: in einigen Einzelwörtern ist es daneben auch üblich, den zweiten Vokal, sofern er akzentuiert ist, mit Glottisschlag neu einzusetzen, z.B. Beate [be' ${ }^{12} \mathrm{a}$ tə] neben [be'a:to], Theater [te' ${ }^{12}$ atte] neben: [te'a:te]. [Hervorhebungen im Original]

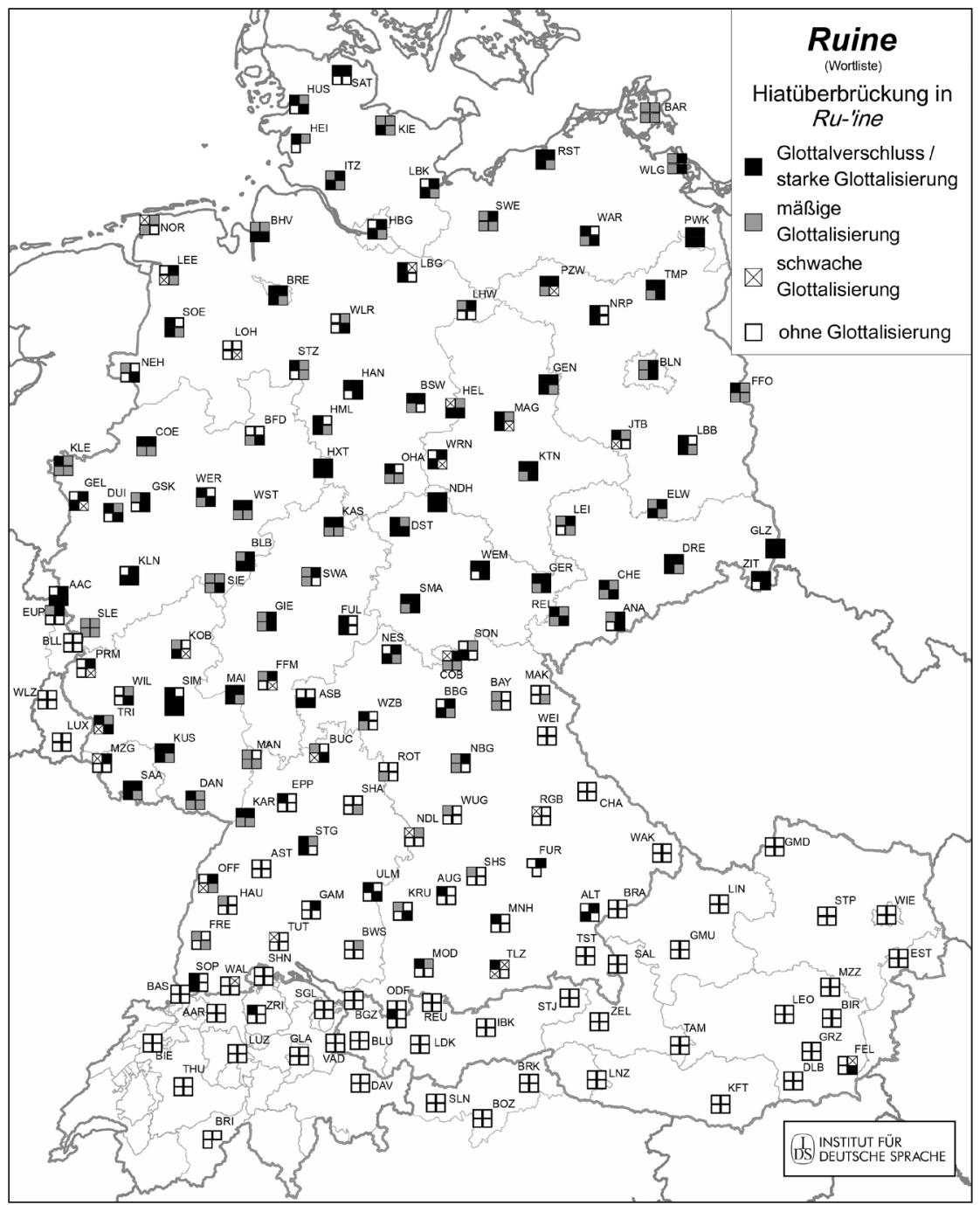

Karte 4: Aussprachevarianten im Hiat in Ruine

Das bedeutet, dass das DAW hier erste Ansätze zur Aufnahme beider Varianten in den Kodex erkennen lässt und damit innovativ ist und die bis dato 
vertretene Ansicht, dass Glottisverschluss im Deutschen nur bei einer Morphemgrenze statthaft sei, anfängt über Bord zu werfen. Allerdings gilt das nur für Beate und Theater, nicht z.B. für Ruine, Oase, real usw.

Beispielhaft für einen Fall einer solchen wortinternen Hiatposition mit betontem zweiten Vokal wird hier das Lexem Ruine untersucht. Auf Karte 4 ist eine klare regionale Staffelung erkennbar: In Mittel- und Norddeutschland ist Glottalisierung die dominierende Variante (83\%). Deutlich seltener wird sie im Süden Deutschlands verwendet (42\%), besonders selten ist sie im Osten Bayerns und im südlichen Baden-Württemberg. In Österreich, in Südtirol, der Schweiz und in Luxemburg kommt die Glottalisierung praktisch gar nicht vor.

Es ist anzunehmen, dass das Phänomen den Sprechern aufgrund der fehlenden graphischen Repräsentation weitgehend unbewusst sein dürfte.

Es liegt damit hier der nicht gerade häufige, aber dafür umso interessantere Fall vor, dass die Minderheit, die die für Deutschland kodifizierte Variante verwendet, vor allem aus Süddeutschland stammt.

\subsection{Quantitätsvariation bei Vokalen}

Dieses Fallbeispiel stammt aus dem Bereich dessen, was man traditionell als Vokalquantität bezeichnet, d.h. den Wechsel zwischen den langen/kurzen bzw. gespannten/ungespannten Vokalpaaren derselben Höhenstufe, die auch mit denselben Vokalgraphemen schriftlich wiedergegeben werden.

Diese Art der Variation tritt standardsprachlich praktisch nur in solchen Fällen auf, wo die Schreibung des Vokals oder die des folgenden Konsonanten keinen eindeutigen Hinweis auf die Quantität des Vokals gibt. Typische Fälle sind darum Ein- und Zweisilbler mit $<\mathrm{ch}>/<$ sch $>$ oder Konsonantengruppen nach dem Vokal (wie z.B. rächen, Dusche, rösten, Wuchs), bei bestimmten Suffixen und Präfixen gibt es Variation, und auch /r/+Konsonant ist ein bekannter Variationskontext. ${ }^{13}$ Nur ganz vereinzelt sind für Deutschland nicht nur innerhalb der entsprechenden phonologischen Distribution, sondern explizit auch beim selben Lexem Varianten kodifiziert (z.B. [i:] und [I] in Dynamit, [y] und [y:] in Nüstern, vgl. DAW, S. 63). ${ }^{14}$

Beispielhaft wird im Folgenden die Vokalquantität in der Präteritalform bing herausgegriffen (die parallel strukturierten Formen fing und ging zeigen im Material grundsätzlich das gleiche sprachgeographische Verhalten). In den Kodizes findet sich dafür nur [hı]] mit kurzem, ungespanntem Vokal, nur für die Schweiz ist auch langes [i:] kodifiziert (Haas/Hove 2009, S. 264).

13 Für einen Überblick über die für diesen Bereich im Gebrauchsstandard vorkommende Variation vgl. König (1989, Bd. 2, S. 147-175).

14 In den Gutachten zur Siebs'schen Regelung sind die Entscheidungen zur Vokalquantität ein mehrfach genannter Kritikpunkt, weil hier häufig nach systematisch-paradigmatischen Gesichtspunkten oder zugunsten norddeutscher Varianten entschieden wurde. 
An dieser Stelle soll mit einem Exkurs potenziellem Sprachgebrauchswandel nachgespürt werden: Werner König hat in den 1970er Jahren für seinen Atlas zur Aussprache des Schriftdeutschen (König 1989) ein strukturell unserem „Deutsch heute“-Material sehr ähnliches Korpus erhoben. Neben den von ihm damals für seinen Atlas ausgewerteten 44 Aufnahmen haben wir an dieser Stelle noch weitere 25 einbezogen, die Werner König damals ebenfalls aufgenommen, aber aus verschiedenen Gründen nicht ausgewertet hat. Sein Korpus ist auf die alten Bundesländer beschränkt.

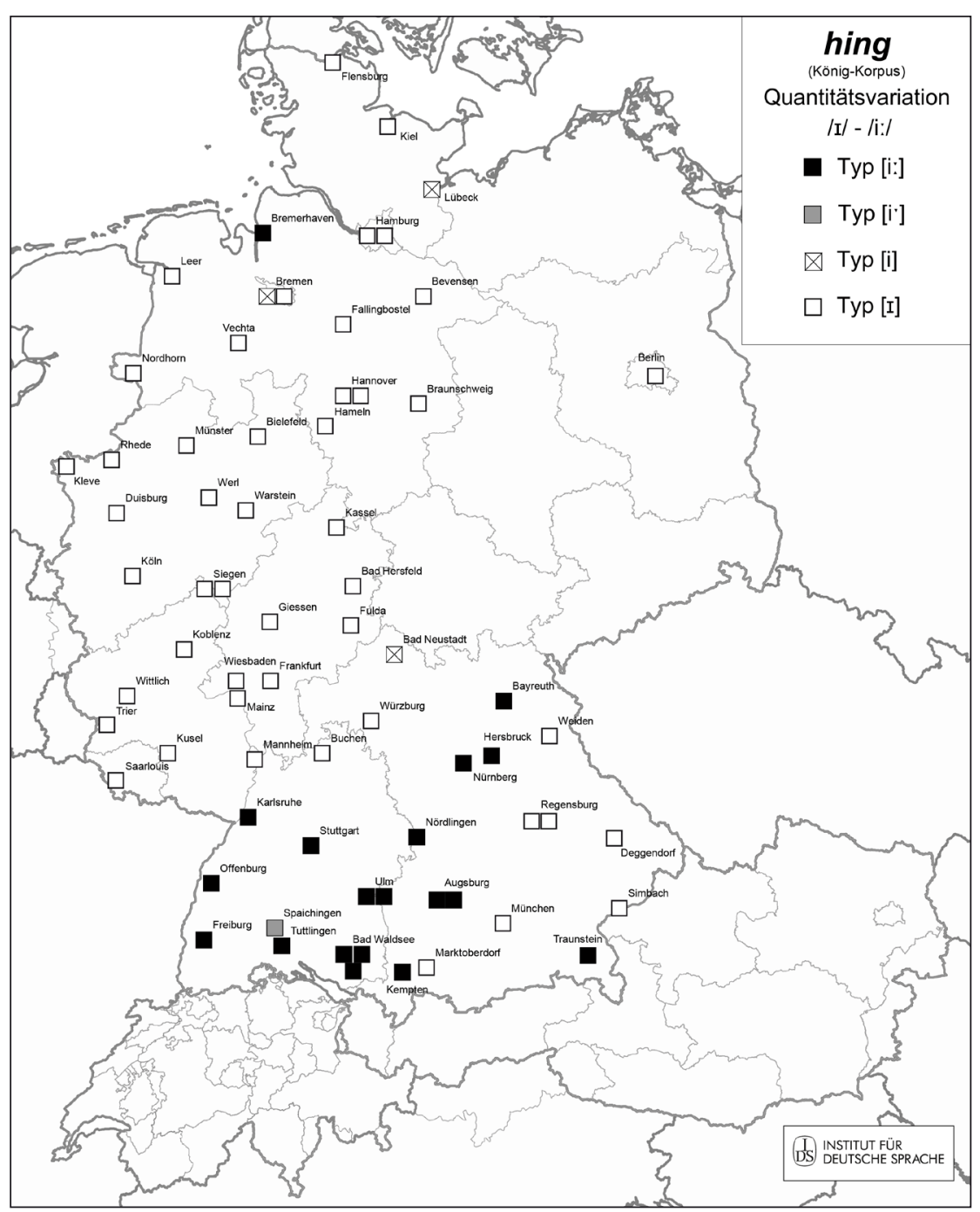

Karte 5: Aussprachevarianten für $<\mathrm{i}>$ in hing im König-Korpus (1976-1977) 


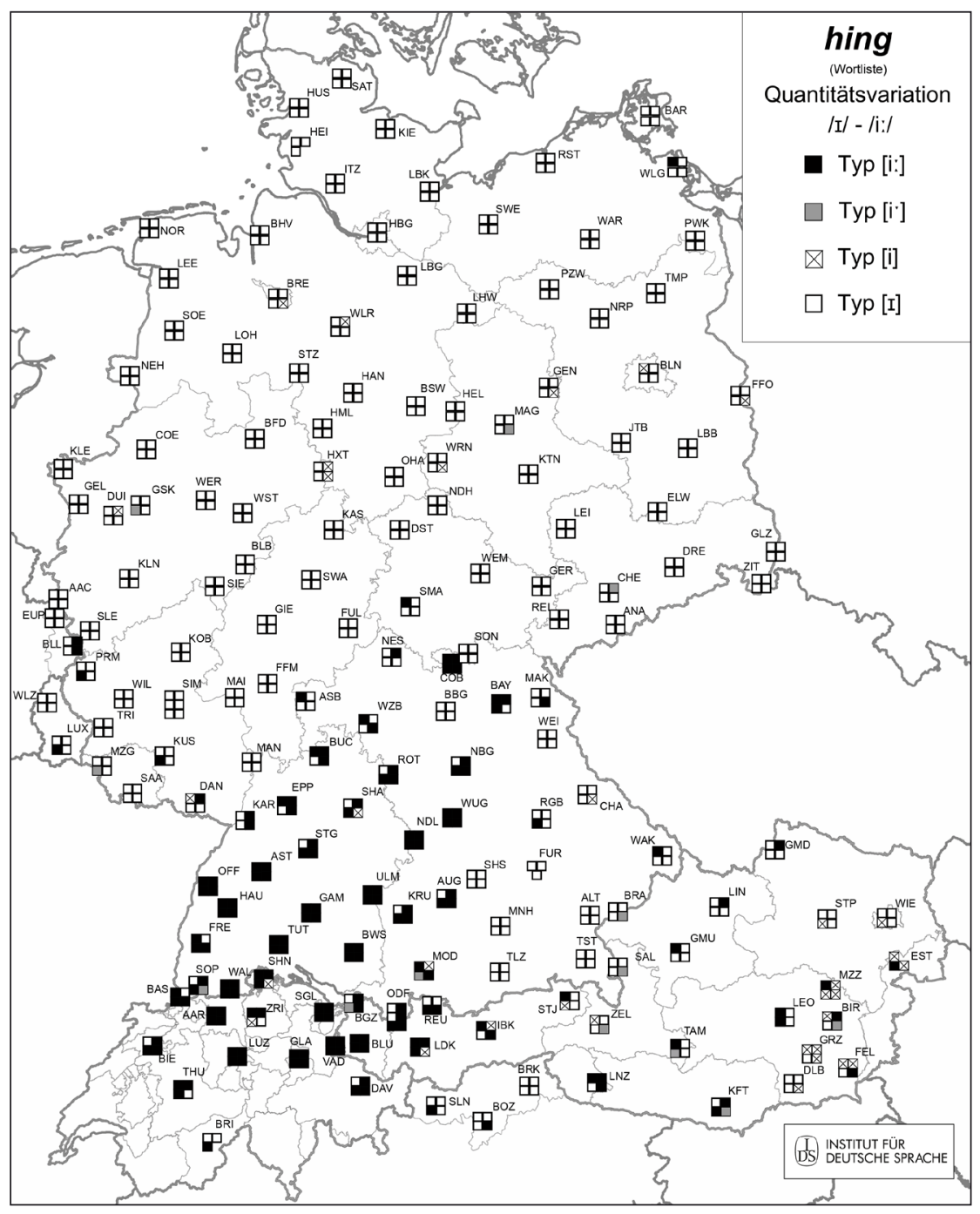

Karte 6: Aussprachevarianten für <i> in bing im Deutsch-heute-Korpus (2006-2009)

Das Kartenbild auf Karte 5 zeigt eine klare regionale Konzentration von [hi:y]-Belegen im Südwesten, d.h. im ganzen alemannischen Sprachraum, und auch in Franken finden sich einige Belege.

Wenn man damit die Befunde aus unserem Korpus auf Karte 6 vergleicht, zeigt sich kaum ein Unterschied, die [hi:n]-Variante hat 30 Jahre später ein praktisch identisches Verbreitungsgebiet, tendenziell scheint sie sich sogar ein wenig ausgebreitet zu haben. Innerhalb des Vorkommensareals weist die hohe Belegdichte auch auf eine hohe Stabilität dieser Form hin. 
Es ist also kein Sprachgebrauchswandel zu Ungunsten der nicht kodifizierten und regional eingeschränkt belegten Form erkennbar. Das ist durchaus nicht selbstverständlich, denn z.B. bei Rache, das ebenfalls im Südwesten traditionell mit Langvokal realisiert wird (vgl. König 1989, Bd. 2, S. 154), ist bei einem analogen real-time-Vergleich ein deutlicher Rückgang der langvokalischen Aussprachevariante festzustellen (von 100\% > 23\%). ${ }^{15}$

Es scheint also sinnvoll, nach einer Begründung für die auffällige Stabilität des Langvokals bei hing zu suchen. Wahrscheinlich spielen Analogien eine wesentliche Rolle: In der Verbalflexion sind Langvokale ein typisches Kennzeichen der Präterita der hochfrequenten starken Verben, die auf einen einfachen Konsonanten enden (kam, lag, schlief, hieß, fiel), so dass durch diese funktionale Analogie wohl auch die Längen in bing, ging und fing (die ja auch untereinander eine Gruppe bilden) gestützt werden.

Auch wenn die Variante [i:] regional klar auf eine süddeutsche Region begrenzt ist, spricht gerade ihre Stabilität dafür, sie bei einer Kodifikation, die den Gebrauchsstandard als Basis hat, zu berücksichtigen. ${ }^{16}$

\subsection{Die Aussprache von $<\mathrm{eu}>/<$ äu $>$}

Ganz anders gelagert und üblicherweise auch nicht als Variationsphänomen diskutiert ist die Aussprache des $<\mathrm{eu}>/<a ̈ u>$ geschriebenen Diphthongs. Es geht hier um das akustisch wenig prominente Zweitglied, genauer darum, ob dieses als gerundeter Vokal vom /ø/- oder /y/-Typus realisiert wird oder als ungerundetes [e] bzw. [I]. Im DAW (S. 73) findet sich dazu die klare Aussage: „Der Zungenrücken [...] wird in einer Gleitbewegung nach vorn [...] verschoben. Die Lippen bleiben gerundet, der Öffnungsgrad des Mundes ändert sich nicht."

Wenn man die anderen Kodizes konsultiert, stößt man - abgesehen von der Höhe des Zweitglieds, dessen Ansatz im mitteltiefen Bereich als [œ] im DAW durchaus kritisch gesehen werden kann - auf dieselbe, einhellige Meinung: Es ist gerundet zu sprechen.

\begin{tabular}{|c|c|c|c|}
\hline Kodex & Transkription & Kodex & Transkription \\
\hline Siebs (Hg.) (1898) & оö & GWDA (1982) & ๑Ø \\
\hline Viëtor (1931) & әу & Duden 6 (2005) & oy \\
\hline Siebs (1969) & ю & DAW (2009) & юе \\
\hline
\end{tabular}

Tab. 1: Kodifizierte Transkriptionen für den $<$ eu $>/<$ äu $>$-Diphthong

$15 \quad$ Vgl. http://prowiki.ids-mannheim.de/bin/view/AADG/RacheQuant.

16 Nach persönlichen Beobachtungen sind die langvokalischen Formen in den drei Präterita auch in den Medien häufig zu hören. 
So eindeutig, wie die Kodizes suggerieren, ist die Lage aber nicht überall in der einschlägigen Literatur. ${ }^{17}$

Otto Bremer stellt in seiner Deutschen Lautlehre (Bremer 1918, S. 34) fest:

Eine dem Buchstabenbilde $e и$ (äu) oder vielmehr dem eines ä̈̈ entsprechende Aussprache gibt es nur in Mecklenburg und in der nördlichen Rheinprovinz. Sonst würde die Schreibung oi unserer Aussprache angemessener sein. [...] der zweite [Bestandteil ist] teils ein kurzes weites $i$, teils ein kurzes $e$.

Auch William Moulton (1962, S. 64 f.) kommt in seinem praxisnahen Buch „The Sounds of English and German“ zu der klaren Feststellung: ,, it is our experience that most German speakers unround their lips during the glide.“

In vergleichbarer Weise wird in der „Einführung in die Phonetik des Deutschen“ (Kohler 1995) und im Phonologiekapitel der Dudengrammatik (Eisenberg 2005) ohne weitere Diskussion mit „,/or/“ ein entrundetes Zweitglied angesetzt.

Der Nachweis ist hier mit signalphonetischen Methoden weit besser zu führen als mittels ohrenphonetischer Transkriptionen. Abbildung 1 zeigt ein auf Messungen der Frequenzen der ersten beiden Vokalformanten beruhendes Diagramm ${ }^{18}$ eines Sprechers aus Hannover, der exemplarisch herausgegriffen wurde, weil er stereotyperweise frei von jeglichem Verdacht ist, regionale Merkmale in seinem Deutsch aufzuweisen.

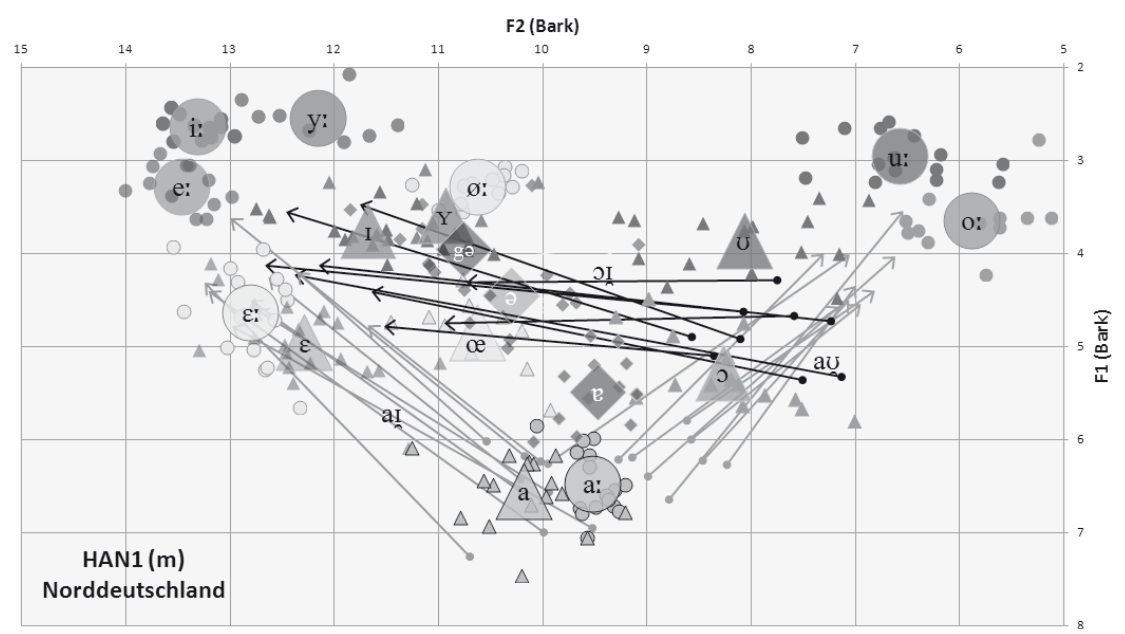

Abb. 1: F1-/F2-Diagramm der Vorleseaussprache (Wortliste) des Sprechers HAN1

17 Beispielhaft zwei Aussagen von Befürwortern: Karl Erbe (1899, S. 183) merkt in seinem Gutachten zur Siebs-Regelung positiv an, dass der Laut nicht als ,breites, unschönes oi, sondern als oö wiedergegeben wird“, und auch die letzte Siebs-Auflage (1969, S. 82) warnt noch davor, das [ø] des zweiten Diphthongteils ,gar mit Entrundung zu [i]“ zu verwandeln.

18 Diese basieren auf insgesamt ca. 250 manuellen Messungen der ersten beiden Vokalformanten aller betonter und einiger unbetonter Vokale. Da diese Prozedur sehr zeitaufwendig ist, kann mit dieser Methode bisher nur exemplarisch gearbeitet werden. 
Im Diagramm werden die gemessenen Mittelwerte der Monophthonge durch große Dreiecke, Kreise und Rauten dargestellt, für die Diphthonge markieren Pfeile die Anfangs- und Endpunkte der einzelnen Belege. Relevant für unsere Fragestellung sind die Endpunkte der schwarzen Pfeile, die für den $<\mathrm{eu}>/<$ äu $>$-Diphthong stehen. Es ist deutlich zu erkennen, dass sie bei diesem Sprecher mehrheitlich durch die Mittelwerte der /œ/- und /Y/-Realisierungen glatt hindurch oder daran vorbeigehen und mehrheitlich im offenen /I/-Bereich enden. Der Diphthong wird also entrundet, eine Wiedergabe mit [or] trifft in diesem Fall die tatsächlichen Gegebenheiten am besten. Vergleichbare Ergebnisse wurden bisher an mehr als 20 Sprecher/innen aus ganz verschiedenen Regionen des deutschen Sprachraums festgestellt. Erste korpusweite Auswertungen, die sowohl auf ohrenphonetischen Transkriptionen als auch auf signalphonetischen Messungen beruhen, zeigen, dass gerundete Zweitglieder in maximal 5\% der Belege auftreten. ${ }^{19}$

\subsection{Die Aussprache von $<y>$}

Kurz erwähnt werden soll auch die Aussprache des Buchstabens $<y>$, genauer, ob er gerundet als [y]- oder ungerundet als [i]-Laut realisiert wird. Die gerundete Aussprache darf als der Normalfall angesehen werden, doch es gibt einige Ausnahmen, unter denen die Aussprache des Ländernamens Libyen einen Spezialfall darstellt, für den alle Kodizes - ähnlich wie bei Embryo - unsilbisches [y] in der Nebensilbe fordern (DAW: [l'iby̆ən]). Unser Korpusbefund zeigt, dass diese Variante nur auf 4\% der Belege kommt, bei den allermeisten besteht eine Art [y]-Metathese (['ly:bion]), d.h. das [y] wird in seine „natürliche“ Position in der Tonsilbe verschoben. Es wären noch einige weitere lexemspezifische Variationsfälle zu nennen: So waren Ägypten und Zylinder im Siebs ursprünglich mit [I] kodifiziert (Siebs 1904, S. 27) und zeigen auch heute noch Variation, ${ }^{20}$ und $[\mathrm{y}]$ in Poly-ist am allerwenigsten wohl in Polyester (DAW: [poly' ${ }^{12}$ Este]) als eine gängige Variante deutschen Gebrauchsstandards anzusehen.

\subsection{Die Aussprache von <-ig>}

Auch die Aussprache des auslautenden <-ig > soll hier noch einmal kurz angerissen werden, weil es eines der bekanntesten Variationsphänomene des Deutschen überhaupt ist. ${ }^{21}$ Die Kodizes fordern für den Auslaut und

\footnotetext{
19 Auch wenn derzeit zu etwaigen regionalen Konzentrationen noch keine endgültigen Ergebnisse vorliegen, sind gerundete Zweitglieder u.a. bei einzelnen Sprechern aus der Generation der 50-60-Jährigen vom Westrand des deutschsprachigen Gebiets (z.B. Eupen) belegt.

20 Der in Nachrichten der letzten Jahre häufig vorkommende Ländername Ägyten wird z.B. in Nachrichtensendungen der ARD konsequent mit [Y] ausgesprochen, im ,heute-journal“ des ZDF hört man dagegen regelmäßig [I].

21 Eine ausführliche Darstellung findet sich in Kleiner (2010).
} 
vor stimmlosem Konsonant bekanntlich einhellig und ausnahmslos die Aussprache mit Frikativ, für die Schweiz und Österreich ist allerdings auch der Plosiv kodifiziert (Haas/Hove 2009, S. 268; Wiesinger 2009, S. 247). Hier zeigt der Korpusbefund grundsätzlich eine klare Nord-Süd-Verteilung, die Frikativaussprache findet sich eher im Norden, die Plosivaussprache eher im Süden des deutschen Sprachraums. Wenn man aber den Befund für Deutschland isoliert betrachtet, zeigen sich im Gebrauchsstandard sehr große Differenzen, die zum Teil lexemspezifisch, vor allem aber positionell bedingt sind. Im prototypischen Lexembeispiel König, also in der Auslautposition, sind 59\% Frikativaussprachen belegt. Vor dem Suffix -keit in Süßigkeiten ist der Frikativ dagegen mit $86 \%$ die weit überwiegende Realisierung. Die Aussprache des Adverbs wenigstens, das eigentlich ein aus dem Paradigma ausgekoppelter Superlativ ist, steht dazu in diametralem Gegensatz, hier haben $92 \%$ der Sprecher in unserem Korpus beim Vorlesen eine plosivische Realisierung verwendet. Trotz dieser empirischen Befunde, die auch mit anderen Belegwörtern klar bestätigen, dass im Auslaut im Vergleich zur Position vor Folgekonsonanz, insbesondere /s/ im Superlativ, eine deutlich abweichende Gebrauchspräferenz besteht, beharren die Kodizes einhellig auf einer Frikativrealisierung. ${ }^{22}$

\section{$4.8<-$ on $>$ in frz. Fremdwörtern}

Als letztes Phänomen soll die Aussprache von auslautendem <-on> in den französischen oder zumindest französisch beeinflussten Lehnwörtern Balkon, Ballon, Eisenbahnwaggon ${ }^{23}$ und Saison thematisiert werden. In Tabelle 2 sind die drei prinzipiell vorkommenden Realisierungstypen [-ว̃:], [-эy] und [-o:n] aufgetragen. Der Korpusbefund für Deutschland zeigt eine ziemlich starke Lexemspezifik: Bei Balkon ist die schriftnahe [-o:n]-Aussprache am häufigsten, bei Ballon und Waggon ist es der Typ mit Velarnasal und bei Saison die am französischen Original orientierte Form mit nasaliertem [-̃̃̃]

Die Kodizes haben dazu eine recht uneinheitliche Meinung: In Siebs (1969) ist der nasalierte Typ, der auch der ursprünglich festgelegten Kodifikation entspricht (die damals [-oy] ausdrücklich verboten hat, vgl. z.B. Siebs 1904, S. 22 f.), durchgängig gebucht, hat aber mit [-o:n] in Balkon und [-oy] in Waggon zusätzlich gerade auch die in unserem Korpus am häufigsten belegten Varianten.

22 Die Realisierung von <-ig > sieht auch Uwe Hollmach, der im Rahmen der Erarbeitung des DAW v.a. die soziophonetischen Akzeptanzuntersuchungen durchgeführt und ausgewertet hat, ,,als kritische orthoepische Frage[n] [...] die erst in folgenden Versuchsreihen zu klären“ sei (Hollmach 2007, S. 292-293).

23 Abgefragt in der Wortliste wurde das Kompositum Eisenbahnwaggon, dessen deutsches Bestimmungswort eine stärker eingedeutschte Aussprache stützen dürfte. In den Aussprachewörterbüchern ist als Vergleichsmöglichkeit nur das Simplex verzeichnet. 


\begin{tabular}{|l|c|c|c|}
\hline \multicolumn{4}{|c|}{ Korpusbefund für Deutschland (in \%) } \\
\hline Lexem & {$[-\tilde{\text { ò }]}$} & {$[-\mathrm{on}]$} & [-o:n] \\
\hline Balkon & 16 & 33 & $\mathbf{4 9}$ \\
\hline Ballon & 36 & $\mathbf{4 6}$ & 18 \\
\hline Eisenbahnwaggon & 39 & $\mathbf{5 4}$ & 7 \\
\hline Saison & $\mathbf{6 1}$ & 35 & 4 \\
\hline
\end{tabular}

Tab. 2: Häufigkeit der Aussprachevarianten [-̃̃:], [-on] und [-o:n] für <-on> in Balkon, Ballon, Eisenbahnwaggon und Saison

Duden (2005) deckt mit seiner Kodifikation bis auf [-o:n] in Saison sogar fast alle im Korpus belegten Varianten ab.

Das DAW schließlich lässt zwar allgemein den [-эy]-Typ zu, bucht aber weder bei Balkon noch bei Saison die in unserem Korpus in Deutschland am häufigsten belegten Varianten und zeigt damit von den drei Kodizes die geringste Schnittmenge mit dem Gebrauchsstandard, wie er bei uns im Korpus belegt ist. ${ }^{24}$

Anzumerken ist, dass die drei belegten Variantentypen natürlich keine homogene Verteilung im deutschen Sprachraum aufweisen, sondern ziemlich klare regionale Präferenzen für die jeweiligen Typen bestehen: [-oy] kommt vor allem im Osten und im Norden Deutschlands vor, [-o:n] im Süden, und auch [-õ:] ist trotz größerer regionaler Streuung nicht gleichmäBig über Deutschland verteilt.25

\section{Zusammenfassung}

Die Relation zwischen der empirisch festgestellten Variation des Gebrauchsstandards und den in den Aussprachewörterbüchern kodifizierten Formen lässt sich grob in drei Typen untergliedern.

1) In Aussprachekodizes für das deutschländische Deutsch sind Formen gebucht, die empirisch im Gebrauchsstandard selten belegbar oder sogar beinahe non-existent sind (z.B. Mikroskop, <eu>/<äu>, Libyen).

2) Weit verbreitete bzw. hochfrequente Varianten sind nicht kodifiziert, und zwar eher typisch süddeutsche (<-ig >, <-on>, hing), aber auch mittel- und norddeutsche (Ruine) und überall in Deutschland gebräuchliche (evangelisch).

$24 \quad$ Wenn man für die vier untersuchten Lexeme die durch die jeweiligen Kodizes abgedeckten Prozentwerte der empirisch belegten Varianten addiert, kommt Duden (2005) auf 396 der maximal möglichen 400 Punkte, Siebs (1969) erreicht 255 und das DAW 220 Punkte.

25 Vgl. die entsprechenden Sprachkarten im AASD (http://prowiki.ids-mannheim.de/bin/view/AADG/ NasalierungOn). 
3) Auch wenn Varianten im Kodex gebucht sind, dann weisen sie im Gebrauchsstandard in vielen Fällen diatopische Variation auf $(<-$ on $>$, Wortakzent $\left.{ }^{20}\right)$.

Die Ursachen für diese Diskrepanzen lassen sich dadurch erklären, dass die deutschen Aussprachekodifikationen

a) am Ideal der Variantenfreiheit festhalten (es soll möglichst nur eine richtige Form geben ${ }^{27}$,

b) an tradierten Formen festhalten (auch an realitätsfernen),

c) einem Systematisierungsdrang folgen: man will konsequente, phonologisch klar gesteuerte Regeln statt lexikalischer Ausnahmen (die das Wörterbuchschreiben natürlich erheblich erschweren, weil man zu deren Feststellung auf eine empirische Basis kaum verzichten kann),

d) eine empirische Ausgangsbasis (sofern überhaupt vorhanden) auf Nachrichtensprecher ,im klassischen Stil“ beschränken,

e) vorhandene Variation (besonders regionale) ausblenden oder gar nicht wahrnehmen,

f) generell eine Konzeption der Standardaussprache für Deutschland ohne sprachliche Regionalität und ohne regional ausgewogene Einbeziehung des Gebrauchsstandards der „educated speaker“ vornehmen.

\section{Alternativen}

Es konnte gezeigt werden, dass die bisher für das Deutsche (in Deutschland) existierenden Aussprachekodizes in vielen Aspekten dem tatsächlich vorhandenen Gebrauchsstandard nicht ausreichend Rechnung tragen. Dieser Zustand ist unbefriedigend, weil dadurch in zahlreichen Fällen Varianten, die bei einer großen Anzahl deutscher Muttersprachler auch in formellsten Situationen gebräuchlich sind, nicht dokumentiert werden. Im Sinne einer realitätsnahen Sprachbeschreibung, die auch häufig vorkommende Varianten erfasst, ist es sinnvoll, primär von sozio-situativen Gebrauchskontexten auszugehen, um Sprachformen Standardsprachcharakter zuzuschreiben (wie es z.B. in der Anglistik auch für die gesprochene Sprache gang und gäbe ist).

$26 \quad$ Wortakzentvariation wurde hier nicht thematisiert, sie ist aber aufgrund der Tatsache, dass Varianten in diesem Bereich auch in den Kodizes relativ häufig dokumentiert sind, ein typischer Fall für diesen dritten Typus. Zur Wortakzentvariation finden sich im AADG zahlreiche Karten.

27 Hier wird eine Auffassung von Standardaussprache vertreten, die „Orthoepie“ als „Rechtlautung“ ganz bewusst parallel setzt zur „Orthographie“/,Rechtschreibung“, obwohl es erhebliche Unterschiede gibt gerade im Bereich der Legitimation (nur die Rechtschreibung hat offizielle Regelungen) und ihrer expliziten Vermittlung (Orthoepie im Sinne einer aktiven Verwendung und Bezugnahme auf Aussprachekodizes spielt im muttersprachlichen Deutschunterricht eine sehr untergeordnete Rolle). 
Mit dieser Voraussetzung als Grundlage sind beispielhaft folgende Alternativen zur bisherigen Kodexschreibung im Bereich der Aussprache des Deutschen denkbar:

1) Die Minimallösung wäre, die im DAW erschienenen Kapitel für Österreich (Wiesinger 2009) und die Schweiz (Haas/Hove 2009) um ein analoges Kapitel für Deutschland zu erweitern. Außerdem sollten alle in diesen Kapiteln beschriebenen Varianten auch ins Wörterverzeichnis übernommen werden (diesen Zustand hatte immerhin schon Siebs 1969).

2) Ebenfalls denkbar wäre eine konzeptionelle Fokussierung in einem spezifischen „Variantenaussprachewörterbuch“, wie es für die Lexik mit dem „Variantenwörterbuch des Deutschen“ seit 2003 existiert (Ammon et al. 2003) ${ }^{28}$ oder für den Bereich der Grammatik in einer „Variantengrammatik des Standarddeutschen ${ }^{\text {(629 }}{ }^{\text {derzeit neu erarbeitet wird. Anders }}$ als diese beiden Projekte, die auf Zeitungskorpora zur Ermittlung der vorhandenen Variation zurückgreifen, müsste für die Ausspracheebene allerdings zuerst genau geplant werden, wie ein Korpus für solche Zwecke sinnvoll stratifiziert sein sollte.

3) Eine Alternative zu Überblicksdarstellungen oder wörterbuchartigen Zusammenstellungen kann auch ein rein deskriptives Informationsangebot bieten, wie es in unserem IDS-Projekt mit Hilfe des „Atlas zur Aussprache des deutschen Gebrauchsstandards“ (AADG) verwirklicht wird.

4) Wenn man ein klassisches Wörterbuch im Stil der bereits existierenden Kodizes neu konzipieren wollte, wäre es sinnvoll, statt eines Mammutwerks mit 150.000 Lemmaeinträgen wie im DAW die Zahl der Stichwörter deutlich zu reduzieren. Gerade im Bereich der fremdsprachigen Toponyme könnte ohne signifikanten Informationsverlust erheblich Platz eingespart werden. Zusammen mit einer ökonomischen lexikografischen Umsetzung (Trunkierungen, Lemmabündelungen, Zeichengröße) könnte dadurch genügend Platz für Varianten und Kommentare freigemacht werden. Der Vorteil wäre, dass man auch hier auf ein bewährtes Vorbild zurückgreifen könnte, denn einen solchen Kodex gibt es schon: Das „Longman Pronunciation Dictionary“ (LPD) von John Wells (Wells 2008), das seit seiner Erstauflage (Wells 1990) mittlerweile in dritter Auflage erschienen ist. Es wirbt ausdrücklich damit, Varianten nicht auszuschließen, sondern explizit zu dokumentieren:

\footnotetext{
28 Eine zweite, völlig neu bearbeitete Auflage befindet sich derzeit in Vorbereitung (vgl. http:// www.variantenwoerterbuch.net, Stand: 19.11.2013).

29 Vgl. www.variantengrammatik.net (Stand: 19.11.2013).
} 
It offers [...]: information on variants [...]. Many English words have a number of different possible pronunciations. [...] Where pronunciations other than the main one are in common educated use, they too are included. (Wells 2008, S. XVII; Hervorhebung im Original)

Weitere Merkmale des LPD sind:

- Konsequente Berücksichtigung von britischem und amerikanischem Englisch im Wörterverzeichnis (nicht nur in Einleitungskapiteln),

- Kommentare und Erläuterungen zu Aussprachevarianten (z.B. semantische Differenzierungen, funktionale Unterschiede),

- Flexionsformen und gängige Ableitungen und Komposita im selben Lemma,

- Grafiken mit Umfrageergebnissen zur Präferenz bestimmter Aussprachevarianten (z.T. mit Differenzierung nach Alter/Region),

- Tonbelege zu allen Lemmata in einer Aussprachedatenbank (d.h. das ganze Wörterbuch ist mit Tonbelegen auf dem Computer installierbar).

Ein vergleichbares Aussprachewörterbuch für das Deutsche, dessen Grundlage zusätzlich noch korpusbasiert sein sollte (das LPD beruht abgesehen von den Umfrageergebnissen - im Wesentlichen auf Expertenwissen), könnte man als optimale Lösung betrachten. Eine Neuauflage des Aussprachewörterbuchs des Duden-Verlags ist meines Wissens schon länger angedacht. Man darf gespannt sein, ob konzeptionelle Veränderungen im Sinne der hier präsentierten Vorschläge vorgenommen werden.

\section{Schluss}

Es war das Anliegen des vorliegenden Beitrags, zu zeigen, dass zwischen den Vorgaben der gängigen deutschen Aussprachekodizes und der tatsächlich verwendeten Standardaussprache, wie sie sich im Korpus „Deutsch heute“ als Gebrauchsstandard von Schülern der gymnasialen Oberstufe präsentiert, oft erhebliche Unterschiede bestehen. Insbesondere sind empirisch im Gebrauchsstandard in der Regel deutlich mehr Varianten belegbar, als sie in den bewusst Varianten ausblendenden deutschsprachigen Aussprachewörterbüchern gebucht sind (zu den Gründen siehe oben).

Um eine möglichst realistische Beschreibung der Aussprache des Deutschen, wie sie im deutschen Sprachraum auch in formellen Situationen gebräuchlich ist, zu dokumentieren, ist eine Erweiterung der als kodifikationsrelevant erachteten Situationen und Sprecher unabdingbar. In diesem Sinne kann das in der Anglistik schon längst etablierte Konzept, den Sprachgebrauch der „educated speakers“ zur Grundlage standardsprachlicher Aus- 
sprachewörterbücher (und Grammatiken) zu machen, ${ }^{30}$ auch für das Deutsche als zeitgemäßer Schritt zu einem erweiterten und damit realitätsnäheren Standardsprachkonzept aufgefasst werden. Interessanterweise ist das Programm dafür schon in Siebs (1969) formuliert, auch wenn es in der Praxis - bis auf die Formen der ,gemäßigten Hochlautung“ - kaum umgesetzt wurde (Siebs 1969, S. 5):

Die gebildete Umgangssprache als eine Abwandlung der Hochsprache wird im sprachlichen Leben unseres Volkes immer wichtiger. Die Umgangssprache auch der meisten Gebildeten (educated speakers") ist gerade in ihrer Lautform mehr oder weniger stark von landschaftlichen Besonderheiten bestimmt.

\section{Literatur}

Ammon, Ulrich (1995): Die deutsche Sprache in Deutschland, Österreich und der Schweiz. Das Problem der nationalen Varietäten. Berlin/New York.

Ammon, Ulrich et al. (2004): Variantenwörterbuch des Deutschen. Die Standardsprache in Österreich, der Schweiz und Deutschland sowie in Liechtenstein, Luxemburg, Ostbelgien und Südtirol. Berlin/New York.

Barbour, Stephen/Stevenson, Patrick (1998): Variation im Deutschen. Soziolinguistische Perspektiven. Berlin u.a.

Behaghel, Otto (1899): Bericht. In: Wissenschaftliche Beihefte zur Zeitschrift des Allgemeinen Deutschen Sprachvereins 16, S. 196-201.

Bremer, Otto (1918): Deutsche Lautlehre. Leipzig.

Brenner, Oskar (1899): Gutachten. In: Wissenschaftliche Beihefte zur Zeitschrift des Allgemeinen Deutschen Sprachvereins 16, S. 178-182.

DAW = Krech, Eva-Maria et al. (2009): Deutsches Aussprachewörterbuch. Mit Beiträgen von Walter Haas, Ingrid Hove, Peter Wiesinger. Unter Mitarbeit von Ines Bose, Uwe Hollmach, Baldur Neuber. Berlin u.a.

Duden (1962): Der große Duden. Bd. 6: Duden Aussprachewörterbuch. Bearb. v. Max Mangold. Mannheim u.a.

Duden (1974): Der große Duden. Bd. 6: Duden Aussprachewörterbuch. Wörterbuch der deutschen Standardaussprache. Bearb. v. Max Mangold in Zusammenarbeit mit der Dudenredaktion. 2., völlig neu bearb. u .erw. Aufl. Mannheim u.a.

Duden (2005): Der Duden. Bd. 6: Duden Aussprachewörterbuch. Bearb. v. Max Mangold in Zusammenarbeit mit der Dudenredaktion. 6., überarb. u. aktual. Aufl. Mannheim u.a.

30 Diese konsequente Orientierung am ,educated speaker“ hat letztlich auch zur Folge, dass im LPD die Demokratisierung der Gesellschaft als Begründung dafür genannt wird, warum man das Konzept der englischen Standardaussprache heutzutage weiter fassen muss: ,, the democratization undergone by English society during the second half of the twentieth century means that it is nowadays necessary to define RP in a rather broader way than was once customary“ (Wells 2008, S. XIX). 
Eisenberg, Peter (2005): Phonem und Graphem. In: Duden. Bd. 4: Die Grammatik. Unentbehrlich für richtiges Deutsch. Hrsg. v.d. Dudenredaktion. 7., völlig neu erarb. u. erw. Aufl. Mannheim u.a., S. 19-94.

Erbe, Karl (1899): Gutachten. In: Wissenschaftliche Beihefte zur Zeitschrift des Allgemeinen Deutschen Sprachvereins 16, S. 182-187.

Goethe, Johann Wolfgang von (1982): Goethes Werke: Hamburger Ausgabe in 14 Bänden. Bd. 12: Schriften zur Kunst. Schriften zur Literatur. Maximen und Reflexionen. Hrsg. v. Erich Trunz. 10. Aufl. München.

GWDA = Krech, Eva-Maria et al. (Hg.) (1982): Großes Wörterbuch der deutschen Aussprache. Leipzig.

Haas, Walter/Hove, Ingrid (2009): Die Standardaussprache in der deutschsprachigen Schweiz. In: DAW, S. 259-277.

Hollmach, Uwe (2007): Untersuchungen zur Kodifizierung der Standardaussprache in Deutschland. (= Hallesche Schriften zur Sprechwissenschaft und Phonetik 21). Frankfurt a.M. u.a.

Kehrein, Roland (2009): Dialektalität von Vorleseaussprache im diatopischen Vergleich - Hörerurteil und phonetische Messung. In: Zeitschrift für Dialektologie und Linguistik 76, S. 14-54.

Kleiner, Stefan (2010): Zur Aussprache von nebentonigem -ig im deutschen Gebrauchsstandard. In: Zeitschrift für Dialektologie und Linguistik 77, S. 259-303.

Kleiner, Stefan (2011): Rezension zu: Eva-Maria Krech/Eberhard Stock/Ursula Hirschfeld/Lutz Christian Anders: Deutsches Aussprachewörterbuch. Mit Beiträgen von Walter Haas, Ingrid Hove, Peter Wiesinger. Unter Mitarbeit von Ines Bose, Uwe Hollmann, Baldur Neuber. In: Zeitschrift für Dialektologie und Linguistik 78, S. 85-91.

Kluge, Friedrich (1899): Gutachten. In: Wissenschaftliche Beihefte zur Zeitschrift des Allgemeinen Deutschen Sprachvereins 16, S. 187-189.

König, Werner (1989): Atlas zur Aussprache des Schriftdeutschen in der Bundesrepublik Deutschland. 2 Bde. Ismaning.

Kohler, Klaus (1995): Einführung in die Phonetik des Deutschen. 2., neubearb. Aufl. (= Grundlagen der Germanistik 20). Berlin.

Moulton, William G. (1962): The sounds of English and German. (= Contrastive Structure Series). Chicago.

Paul, Hermann (1899): Gutachten. In: Wissenschaftliche Beihefte zur Zeitschrift des Allgemeinen Deutschen Sprachvereins 196, S. 189-191.

Schmidt, Jürgen E./Herrgen, Joachim (2011): Sprachdynamik: Eine Einführung in die moderne Regionalsprachenforschung. (= Grundlagen der Germanistik 49). Berlin.

Siebs, Theodor (Hg.) (1898): Deutsche Bühnenaussprache. Berlin u.a.

Siebs, Theodor (1904): Grundzüge der Bühnenaussprache. 2. Aufl. Berlin u.a.

Siebs, Theodor (1931a): Rundfunkaussprache. Berlin. 
Siebs, Theodor (1931b): Deutsche Bühnenaussprache. Hochsprache. Köln.

Siebs, Theodor (1969): Deutsche Aussprache. Reine und gemäßigte Hochlautung mit Aussprachewörterbuch. Hrsg. v. Helmut de Boor, Hugo Moser u. Christian Winkler. 19., umgearb. Aufl. Berlin.

Trouvain, Jürgen (2011): Rezension zu: Krech, Eva-Maria; Stock, Eberhard; Hirschfeld, Ursula \& Anders, Lutz Christian 2009. Deutsches Aussprachewörterbuch. Berlin, New York: de Gruyter. In: Zeitschrift für interkulturellen Fremdsprachenunterricht 16, 1, S. 86-91.

Viëtor, Wilhelm (1885): Die Aussprache der in dem Wörterverzeichnis für die deutsche Rechtschreibung zum Gebrauch in den preussischen Schulen enthaltenen Wörter. Heilbronn.

Viëtor, Wilhelm (1931): Deutsches Aussprachewörterbuch. 4. u. 5., durchges. u. durch e. Anhang erw. Aufl. Besorgt von Ernst A. Meyer. Leipzig.

WDA = Krech, Hans et al. (Hg.) (1964): Wörterbuch der deutschen Aussprache. Leipzig.

Wells, John C. (1990): Longman Pronunciation Dictionary. 1. Aufl. Harlow.

Wells, John C. (2008): Longman Pronunciation Dictionary. 3. Aufl. Harlow.

Wiesinger, Peter (2009): Die Standardaussprache in Österreich. In: DAW, S. 229-258. 